\title{
Steroids in airway diseases (summary)
}

\author{
T. J. H. Clark \\ B.Sc., M.D., M.R.C.P.
}

Cardiothoracic Institute, University of London

CORTICOSTEROIDS are of particular value in the treatment of asthma and they can be used in two main clinical circumstances-for acute severe asthma and for control of chronic asthma.

There is no good evidence showing that corticosteroids materially influence the natural history of acute severe asthma but the treatment is widely used in the hope that death is averted and recovery hastened. Patients with asthma do not metabolize corticosteroids in a unique manner and to maintain a plasma cortisol of over $100 \mu \mathrm{g} \%$ about $3 \mathrm{mg} / \mathrm{kg}$ of i.v. hydrocortisone hemisuccinate is required approximately every $6 \mathrm{hr}$. Previous treatment with oral corticosteroids does not alter the dose required. Adrenal responsiveness appears normal in patients with asthma who have not been treated with systemic corticosteroids, and adrenal stimulants such as ACTH can be used with success. Patients appear to take $6-8 \mathrm{hr}$ before a response to i.v. treatment is evident.

Systemic corticosteroid treatment for chronic asthma has been used successfully for many years but is frequently associated with many unwanted side effects. Recently, aerosols containing corticosteroids have been used to avoid these systemic effects and there is good evidence that given by inhalation steroids act topically. Inhalation of corticosteroids has increasingly become the treatment of choice for patients with chronic asthma not responding to bronchodilators and sodium cromoglycate, and the only side effect of note appears to be oral candidiasis. This complication is dose-related and rarely causes symptoms and is thought by most clinicians to be a lesser evil than systemic corticosteroids. 